\title{
Relativistic description of the photoelectric effect
}

\author{
Julio Güémez* \\ Department of Applied Physics, Faculty of Sciences, \\ University of Cantabria, Av. de Los Castros, 39005 Santander, Spain \\ Manuel Fiolhais ${ }^{\dagger}$ \\ Physics Department and CFisUC, University of Coimbra, 3004-516 Coimbra, Portugal
}

(Dated: June 6, 2018)

\begin{abstract}
Einstein's famous explanation of the photoelectric effect is addressed using the Minkowski fourvector formalism. This formalism deals with energy and linear momentum simultaneously and it is Lorentz covariant. The energy and momentum equations for both electron emission and electron braking are obtained in the lab frame, S. It is then shown that the energy and momentum equations for electron emission and electron braking in a second inertial frame, $\mathrm{S}^{\prime}$, are linear combinations of the corresponding equations in the original frame, S, according to the principle of relativity. Although the photoelectric effect is basically a non-relativistic phenomenon, the approach presented here may provide new insights for both instructors and students.
\end{abstract}




\section{INTRODUCTION}

The photoelectric effect is the emission of electrons from the illuminated surface of a metal. ${ }^{1}$ To escape from the surface, an electron must absorb enough energy from the incident light to overcome the net attractive forces produced by the rest of the material. ${ }^{2}$ Thus, the electron must overcome a potential-energy barrier and the light supplies the required energy for the electron to escape. ${ }^{3}$

The photoelectric effect is presented in introductory courses, ${ }^{4}$ in inservice teacher training, ${ }^{5}$ in demonstrations, ${ }^{6,7}$ and in undergraduate lab classes, to measure the ratio $h / e$ and Planck's constant, $h .^{8-10}$

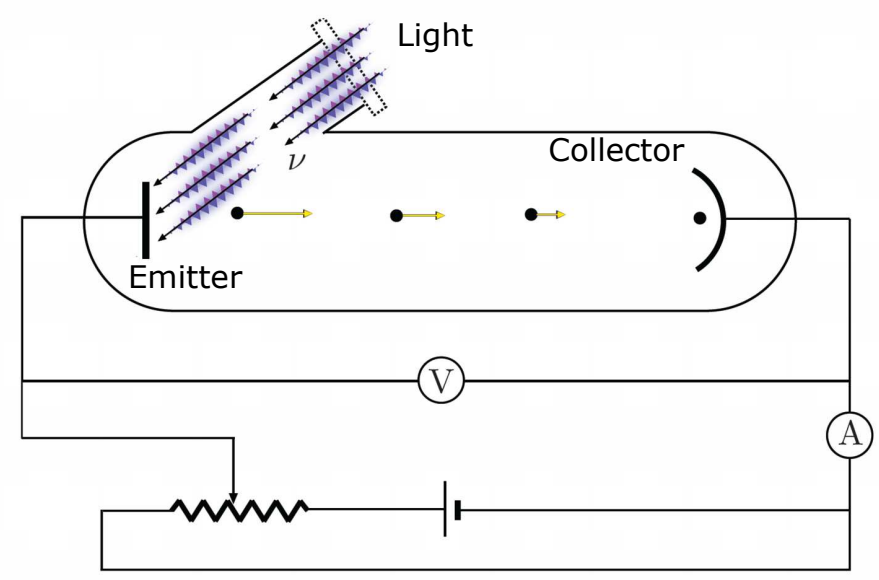

FIG. 1. Typical photoelectric effect circuit with a photocell, a potentiometer, a voltmeter, and an ammeter.

The typical photoelectric experiment ${ }^{11,12}$ makes use of an electric circuit with a photocell (The cathode, or emitter, is usually a polished metal plate inside an evacuated chamber.), a potentiometer, a voltmeter, and an ammeter (Fig. 1). When light with sufficiently high frequency, $\nu$, is directed to the polished metal, electrons are ejected from it. With the potentiometer, an electric potential difference, $V$, can be maintained between the metal surface and a collector cup (the anode) to sweep up these electrons, known as photoelectrons. ${ }^{13}$ Then, a current, which can be measured with an ammeter, flows in the circuit. It is also possible to slow down the ejected electrons by adjusting the potential difference. A minimum voltage known as the stopping potential, ${ }^{14} V_{\min }$, exists for which the ammeter reading drops to zero. In such case, no photoelectrons reach the anode. 
The existence of the photocurrent depends on the frequency of the light. For a given metal, monochromatic light with a frequency below the threshold frequency, $\nu_{\mathrm{th}}$, produces no photocurrent, regardless of the intensity of the light. ${ }^{6}$ Moreover, when the photoelectric effect takes place, there is a very small time delay, difficult to be measured, between the light absorption and the photoelectron emission. ${ }^{15}$ In addition, the stopping potential does not depend on the light intensity, but does depend on the light frequency. ${ }^{16}$ The emitted electrons for a given frequency, $\nu>\nu_{\text {th }}$, have a maximum kinetic energy, $K_{\max }(\nu)$, resulting in a maximum speed, $v_{\max }(\nu)$.

Albert Einstein was awarded the 1921 Nobel Prize in physics for his explanation of this effect, ${ }^{17}$ which cannot be understood within the restricted framework of classical physics.

Textbooks usually deal with the photoelectric effect by means of the energy balance equation

$$
h \nu-\Phi=\frac{1}{2} m_{\mathrm{e}} v_{\max }^{2}(\nu),
$$

where $m_{\mathrm{e}}$ is the electron mass and $\Phi$ is the work function. ${ }^{18}$ The maximum kinetic energy of the photoelectrons is related to the minimum voltage required to prevent the electrons from reaching the anode, resulting in zero current circulating throughout the circuit:

$$
e V_{\min }(\nu)=K_{\max }(\nu)=\frac{1}{2} m_{\mathrm{e}} v_{\max }^{2}(\nu),
$$

where $e$ is the elementary charge. Combining Eqs. (1) and (2), one obtains ${ }^{19}$

$$
e V_{\min }(\nu)=h \nu-\Phi
$$

This equation, except for the notation, is the very same that Einstein wrote in his 1905 article $^{20}$ where the concept of light quantum — the photon, after G. N. Lewis ${ }^{21}$ — was introduced. Einstein himself proposed ${ }^{20}$ the experimental setup with the noted electric circuit to test this theory, specifically Eq. (3), and the experiment was performed by R. Millikan in 1916. ${ }^{11,22,23}$ The graphical representation of the minimum voltage as a function of the frequency is a straight line with a slope $h / e$, irrespective of the metal. ${ }^{12}$ The subsequent straight line crosses the horizontal axis at the threshold frequency, hence $\Phi=h \nu_{\text {th }}$ (Fig. 2).

The previous equations and discussion give rise to the following remarks:

1. In 1916, Einstein established that the light quantum ought to have linear momentum. ${ }^{24}$ Specifically, a photon with energy $E=h \nu$ has a linear momentum of magnitude 


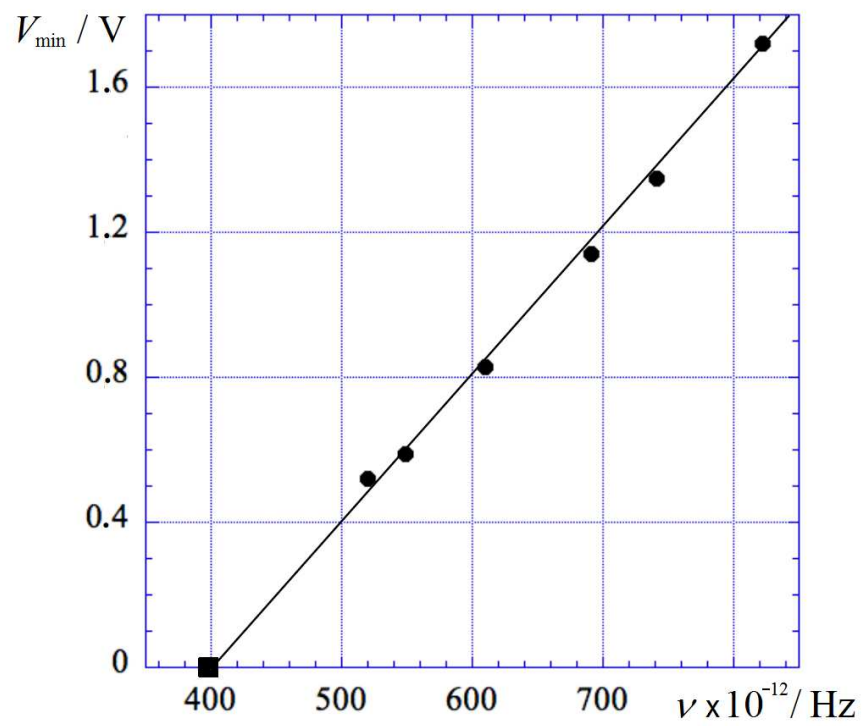

FIG. 2. Minimum voltage $V_{\min }$ (in volts) as a function of the frequency $\nu$ (adapted from Ref. 12). The straight line, with slope $h / e=(3.98 \pm 0.18) \times 10^{-15} \mathrm{~V}$ s, crosses the horizontal axis at the threshold frequency, $\nu_{\mathrm{th}}=(398 \pm 18) \times 10^{12} \mathrm{~Hz}$. The source for this data is also Ref. 12 .

$p=h \nu / c$. In Eq. (1) there is no reference to the linear momentum balance in the process.

2. Equations (1) and (3) are not covariant under Galilean transformations, even including the Doppler effect change of the frequency, or under Lorentz transformations, including the relativistic transformations for velocities and frequencies.

Interestingly enough, the photoelectric effect offers the possibility for an analysis of a physical process in which relativity (The description must be Lorentz invariant.), quantum physics (photon as the quantum of the electromagnetic field), and thermodynamics (work function as internal energy) play a role. ${ }^{25}$ Therefore, it appears that the relativistic description allows for a deeper understanding of the relevant physical concepts of those three areas: relativity, quantum physics, and thermodynamics. ${ }^{5}$

Even college-level physics textbooks that introduce a more modern perspective, ${ }^{26}$ especially in regards to mechanics with connections to quantum mechanics and relativity, deal with the photoelectric effect in the traditional way. To the extent of our knowledge, a relativistic description of the photoelectric effect was presented in 1931 and 1933 papers in Physical Review by Hall and Oppenheimer, ${ }^{27,28}$ but these articles were not intended to be 
and are not pedagogical. In the present article, the four-vector formalism of relativity is used. This formalism is a powerful tool, since it is fully covariant under Lorentz transformations, and it simultaneously deals with the energy and linear momentum conservation in an

elegant fashion. When a student masters this formalism, he/she may find it enlightening to re-visit the photoelectric effect, in order to enjoy the conceptual and pedagogical advantages of the approach described in this article.

This paper is organized as follows. In Section II the four-vector description of the photoelectric effect is introduced, and in Section III the process is described in another inertial frame. In Section IV conclusions are drawn, focusing on the pedagogical advantages of describing the effect within a relativistic formalism.

\section{FOUR-VECTOR DESCRIPTION}

Initially, the metal plate is taken to be at rest in the lab frame. The mass of the metal plate is finite. Therefore, the metal plate acquires a non-zero recoil velocity as a consequence of photon absorption and electron emission. The limit of infinite plate mass and zero recoil velocity will be discussed shortly.

In the inertial reference frame, $\mathrm{S}$ (lab frame), the frequency of the incident light is $\nu$. Thus the energy and linear momentum of the photon are $h \nu$ and $(-h \nu / c, 0,0)$, respectively, where the photon is propagating in the negative $x$ direction. The four-vector momentum-energy for the photon is thus given by

$$
\mathrm{E}_{\mathrm{ph}}=\left(\begin{array}{c}
-c(h \nu / c) \\
0 \\
0 \\
h \nu
\end{array}\right)
$$

Briefly consider the inverse photoelectric effect, i.e., the process in which initially the metal plate is missing an electron, and then absorbs an electron and emits a photon. The plate's initial internal energy, $E_{\mathrm{i}}$, at temperature $T$, can be written as

$$
E_{\mathrm{i}}=E_{0}+m_{\mathrm{e}} c^{2}-\Phi
$$

where

$$
E_{0}=\sum_{j} m_{j} c^{2}-\tilde{U}+U(T)
$$


Here, $m_{j}$ is the mass of the $j$-th elementary particle that forms the metal plate, $\tilde{U}$ is the total binding energy, $U(T)$ is the part of the internal energy related to temperature, and $E_{0}$ is the plate's internal energy without one electron. The electron is taken to be at rest before it enters the metal plate. When the plate captures the electron, its energy increases by $m_{\mathrm{e}} c^{2}$, aside from the change in the binding energy produced by the interaction of the electron with the rest of the plate, which is taken into account in the value of $\Phi$. The plate then emits a photon with the threshold frequency $\nu_{\mathrm{th}}$. Therefore, $\Phi=h \nu_{\mathrm{th}}$.

After the plate has undergone the photoelectric effect, i.e., after it has absorbed a photon with $\nu=\nu_{\text {th }}$ and emitted the electron, its internal energy is

$$
E_{\mathrm{f}}=E_{\mathrm{i}}-m_{\mathrm{e}} c^{2}+\Phi=E_{0} .
$$

If $\nu>\nu_{\mathrm{th}}$, the difference between the incident photon energy and the threshold energy goes into kinetic energy of the emitted electron (with maximum speed, $v_{\max }(\nu)$ ); of course, if $\nu=\nu_{\mathrm{th}}$, then the velocity of the emitted electron vanishes.

The initial and final momentum-energy four-vectors for the plate are given by

$$
\mathrm{E}_{\mathrm{i}}=\left(\begin{array}{c}
0 \\
0 \\
0 \\
E_{\mathrm{i}}
\end{array}\right), \quad \mathrm{E}_{\mathrm{f}}=\left(\begin{array}{c}
-c \gamma\left(v_{\mathrm{f}}\right) M_{0} v_{\mathrm{f}} \\
0 \\
0 \\
\gamma\left(v_{\mathrm{f}}\right) E_{0}
\end{array}\right) \text {, }
$$

respectively, where

$$
\gamma(v)=\left[1-\beta^{2}(v)\right]^{-1 / 2}
$$

and

$$
\beta(v)=\frac{v}{c}
$$

The second four-vector corresponds to the plate with inertia $M_{0}=c^{-2} E_{0}$, moving in the negative $x$ direction with velocity $\vec{v}_{\mathrm{f}}=\left(-v_{\mathrm{f}}, 0,0\right)$.

The momentum-energy four-vector of the electron emitted with maximum speed, $v_{\max }$, in the positive $x$ direction is given by

$$
\mathrm{E}_{\mathrm{e}}=\left(\begin{array}{c}
c \gamma\left(v_{\max }\right) m_{\mathrm{e}} v_{\max } \\
0 \\
0 \\
\gamma\left(v_{\max }\right) m_{\mathrm{e}} c^{2}
\end{array}\right)
$$

Here and henceforth, $v_{\max }$ means $v_{\max }(\nu)$. 


\section{A. Electron emission}

The momentum-energy four-vector equation for the process is given by

$$
E_{f}+E_{e}=E_{i}+E_{p h},
$$

which leads to the following equations:

$$
\gamma\left(v_{\mathrm{f}}\right) M_{0} v_{\mathrm{f}}=\gamma\left(v_{\max }\right) m_{\mathrm{e}} v_{\max }+c^{-1} h \nu,
$$

and, with the use of Eq. (5) and $M_{0}=c^{-2} E_{0}$,

$$
h \nu-\Phi=\left[\gamma\left(v_{\mathrm{f}}\right)-1\right] M_{0} c^{2}+\left[\gamma\left(v_{\max }\right)-1\right] m_{\mathrm{e}} c^{2} .
$$

From these two equations one obtains $v_{\mathrm{f}}$ and $v_{\max }$. If the photoelectron is emitted with a speed smaller than $v_{\max }$, the energy difference remains in the plate as internal energy.

When a photon hits the plate and is absorbed, with emission of an electron, in a process that occurs in a small, but non-zero, time interval, $\Delta t$, there is an average non-zero and finite force acting on the plate with magnitude denoted by $F$. The corresponding impulse on the plate has a magnitude of $F \Delta t$, and thus

$$
\gamma\left(v_{\mathrm{f}}\right) M_{0} v_{\mathrm{f}}=F \Delta t .
$$

In the limit of infinite mass for the plate, $v_{\mathrm{f}} \rightarrow 0$. Since $\lim _{M_{0} \rightarrow \infty} \gamma\left(v_{\mathrm{f}}\right) M_{0} v_{\mathrm{f}}$ is finite and, for $v_{\mathrm{f}}^{2} / c^{2} \ll 1$, one has $\gamma\left(v_{\mathrm{f}}\right) \rightarrow 1+\frac{1}{2} \frac{v_{\mathrm{f}}^{2}}{c^{2}}$, it follows that

$$
\lim _{v_{\mathrm{f}}^{2} / c^{2} \ll 1}\left[\gamma\left(v_{\mathrm{f}}\right)-1\right] M_{0} c^{2} \rightarrow \frac{1}{2}\left(M_{0} v_{\mathrm{f}}\right) v_{\mathrm{f}},
$$

and so

$$
\lim _{M_{0} \rightarrow \infty, v_{\mathrm{f}} \rightarrow 0}\left[\gamma\left(v_{\mathrm{f}}\right)-1\right] M_{0} c^{2}=0 .
$$

In this limit, the plate remains at rest, so that the force acting on it does not perform any work, and the equations describing the process are

$$
\begin{aligned}
c^{-1} h \nu & =-\gamma\left(v_{\max }\right) m_{\mathrm{e}} v_{\max }+F \Delta t \\
h \nu & =\left[\gamma\left(v_{\max }\right)-1\right] m_{\mathrm{e}} c^{2}+\Phi .
\end{aligned}
$$

Note that Eq. (18) is valid generally, but Eq.(19) is valid only in the limit $M_{0} \rightarrow \infty$. It is evident that the plate experiences the necessary impulse to absorb the photon and emit the electron with velocity $v_{\max }$. In the limit $v_{\max }^{2} / c^{2} \ll 1$, which is required by $M_{0} \rightarrow \infty$, Eq. (19) reduces to Eq. (1). 


\section{B. Electron braking}

Suppose that in the photoelectric experiment, one omits the potentiometer and instead places the entire apparatus between the plates of a capacitor. Then, the velocity of the

electron is altered by the electric force generated by the electric field, $\overrightarrow{\mathcal{E}}$, established between the plates of the capacitor. The momentum-energy conservation relation is given by the equation

$$
\mathrm{E}_{\mathrm{e}, \mathrm{f}}-\mathrm{E}_{\mathrm{e}, \mathrm{i}}=\mathrm{W}
$$

where $E_{e, i}\left(E_{e, f}\right)$ is the initial (final) electron momentum-energy four-vector for this braking process and $\mathrm{W}^{\mu}=-e \int \mathrm{F}_{\nu}^{\mu} \mathrm{dx}^{\nu}$ is the $\mu$-th component of the electron impulse-work fourvector $^{29}$ transferred to the electron. Here, $\mathrm{F}_{\nu}^{\mu}$ are the components of the electromagnetic field tensor ${ }^{30}$ and $\mathrm{dx}^{\nu}$ are the components of the electron infinitesimal displacement four-vector. In the present case, there exists only a non-vanishing $x$-component for the electromagnetic field, namely $\mathcal{E}_{x}=\mathcal{E}$, which is a constant. Therefore

$$
\mathrm{W}=-e\left(\begin{array}{cccc}
0 & 0 & 0 & \mathcal{E} \\
0 & 0 & 0 & 0 \\
0 & 0 & 0 & 0 \\
\mathcal{E} & 0 & 0 & 0
\end{array}\right)\left(\begin{array}{c}
L \\
0 \\
0 \\
c t_{0}
\end{array}\right)=-e\left(\begin{array}{c}
c \mathcal{E} t_{0} \\
0 \\
0 \\
\mathcal{E} L
\end{array}\right)
$$

Here $t_{0}$ is the electron's time of flight and $L$ is the distance traveled by the electron.

Explicitly, for an electron emitted with maximum speed that stops just before reaching the negative plate, Eq. (20) reads

$$
\left(\begin{array}{c}
0 \\
0 \\
0 \\
m_{\mathrm{e}} c^{2}
\end{array}\right)-\left(\begin{array}{c}
c \gamma\left(v_{\max }\right) m_{\mathrm{e}} v_{\max } \\
0 \\
0 \\
\gamma\left(v_{\max }\right) m_{\mathrm{e}} c^{2}
\end{array}\right)=-e\left(\begin{array}{c}
c \mathcal{E}_{\min } t_{0} \\
0 \\
0 \\
\mathcal{E}_{\min } L
\end{array}\right)
$$

Here $\mathcal{E}_{\text {min }}$ is the minimum electric field magnitude required to stop the maximum speed photoelectrons just before they reach the anode. This four-vector equation leads to

$$
e \mathcal{E}_{\min } t_{0}=\gamma\left(v_{\max }\right) m_{\mathrm{e}} v_{\max }
$$

and

$$
e \mathcal{E}_{\min } L=\left[\gamma\left(v_{\max }\right)-1\right] m_{\mathrm{e}} c^{2} .
$$


In the four-vector formalism, the equation describing the whole process - i.e. from light absorption to electron stopping - states that the global energy variation of the system equals the impulse-work transferred to it:

$$
\left(E_{\mathrm{f}}+\mathrm{E}_{\mathrm{e}, \mathrm{f}}\right)-\left(\mathrm{E}_{\mathrm{i}}+\mathrm{E}_{\mathrm{ph}}\right)=\mathrm{W} .
$$

All these vectors are defined above, and one readily obtains:

$$
\left(\begin{array}{c}
-c F \Delta t \\
0 \\
0 \\
E_{0}
\end{array}\right)+\left(\begin{array}{c}
0 \\
0 \\
0 \\
m_{\mathrm{e}} c^{2}
\end{array}\right)-\left(\begin{array}{c}
0 \\
0 \\
0 \\
E_{0}+m_{\mathrm{e}} c^{2}-\Phi
\end{array}\right)-\left(\begin{array}{c}
-h \nu \\
0 \\
0 \\
h \nu
\end{array}\right)=-e\left(\begin{array}{c}
c \mathcal{E}_{\min } t_{0} \\
0 \\
0 \\
\mathcal{E}_{\min } L
\end{array}\right) .
$$

With the use of Eq. (18) one arrives at

$$
\gamma\left(v_{\max }\right) m_{\mathrm{e}} v_{\max }=e \mathcal{E}_{\min } t_{0},
$$

and

$$
h \nu-\Phi=e \mathcal{E}_{\min } L .
$$

Since $\mathcal{E}_{\min } L=V_{\min }$, Eq. (28) is equivalent to Eq. (3).

\section{DESCRIPTION IN ANOTHER INERTIAL FRAME}

According to the relativity principle, in the reference frame $\mathrm{S}^{\prime}$, in standard configuration moving with velocity $\vec{u}$ in the $x$-direction with respect to $\mathrm{S}$, the four-vector Eqs. (12) and (25) are replaced by

$$
\mathrm{E}_{\mathrm{f}}^{\prime}+\mathrm{E}_{\mathrm{e}}^{\prime}=\mathrm{E}_{\mathrm{i}}^{\prime}+\mathrm{E}_{\mathrm{ph}}^{\prime},
$$

and

$$
\left(\mathrm{E}_{\mathrm{f}}^{\prime}+\mathrm{E}_{\mathrm{e}, \mathrm{f}}\right)-\left(\mathrm{E}_{\mathrm{i}}^{\prime}+\mathrm{E}_{\mathrm{ph}}^{\prime}\right)=\mathrm{W}^{\prime},
$$

respectively. Here

$$
\mathrm{E}_{\mathrm{f}}^{\prime}=\mathcal{L}(\vec{u}) \mathrm{E}_{f},
$$

and similarly for the other four-vectors, where the Lorentz transformation matrix is given by 


$$
\mathcal{L}(\vec{u})=\left(\begin{array}{cccc}
\gamma(u) & 0 & 0 & -\gamma(u) \beta(u) \\
0 & 1 & 0 & 0 \\
0 & 0 & 1 & 0 \\
-\gamma(u) \beta(u) & 0 & 0 & \gamma(u)
\end{array}\right) .
$$

Let us consider the photon absorption/electron emission process, described by Eq. (12), which can be written as

$$
E_{f}-E_{i}=E_{p h}-E_{e},
$$

in S. In the limit $M_{0} \rightarrow \infty$, the left-hand side of this equation reads

$$
\mathrm{E}_{\mathrm{f}}-\mathrm{E}_{\mathrm{i}}=\left(\begin{array}{c}
-c F \Delta t \\
0 \\
0 \\
E_{0}
\end{array}\right)-\left(\begin{array}{c}
0 \\
0 \\
0 \\
E_{0}+m_{\mathrm{e}} c^{2}-\Phi
\end{array}\right)=\left(\begin{array}{c}
-c F \Delta t \\
0 \\
0 \\
\Phi-m_{\mathrm{e}} c^{2}
\end{array}\right) .
$$

From Eqs. (31), (32) and (34), it follows that

$$
\mathrm{E}_{\mathrm{f}}^{\prime}-\mathrm{E}_{\mathrm{i}}^{\prime}=\left(\begin{array}{c}
-\gamma(u) c F \Delta t-\gamma(u) \beta(u)\left(\Phi-m_{\mathrm{e}} c^{2}\right) \\
0 \\
0 \\
\gamma(u) \beta(u) c F \Delta t+\gamma(u)\left(\Phi-m_{\mathrm{e}} c^{2}\right)
\end{array}\right) .
$$

Similarly, from Eqs (4), (11), (31), and (32) one has

$$
\mathrm{E}_{\mathrm{ph}}^{\prime}-\mathrm{E}_{\mathrm{e}}^{\prime}=\left(\begin{array}{c}
-h \nu \gamma(u)[1+\beta(u)]-c \gamma(u) \gamma\left(v_{\max }\right) m_{\mathrm{e}}\left[v_{\max }-c \beta(u)\right] \\
0 \\
0 \\
h \nu \gamma(u)[1+\beta(u)]-\gamma(u) \gamma\left(v_{\max }\right)\left[1-v_{\max } \beta(u) / c\right] m_{\mathrm{e}} c^{2}
\end{array}\right) .
$$

Equations (31), (33), (35), and (36) leads to the following pair of equations:

$$
\begin{aligned}
\gamma(u) c F \Delta t+\gamma(u) \beta(u)\left(\Phi-m_{\mathrm{e}} c^{2}\right)= & h \nu \gamma(u)[1+\beta(u)] \\
& +c \gamma(u) \gamma\left(v_{\max }\right) m_{\mathrm{e}}\left[v_{\max }-c \beta(u)\right]
\end{aligned}
$$

and

$$
\begin{aligned}
\gamma(u) \beta(u) c F \Delta t+\gamma(u)\left(\Phi-m_{\mathrm{e}} c^{2}\right)= & h \nu \gamma(u)[1+\beta(u)] \\
& -\gamma(u) \gamma\left(v_{\max }\right)\left[1-v_{\max } \beta(u) / c\right] m_{\mathrm{e}} c^{2} .
\end{aligned}
$$


The energy equation (38) can be written as $\gamma(u) \beta(u) c \times$ Eq. (18) $+\gamma(u) \times$ Eq. (19), namely

$$
\left\{\begin{aligned}
\gamma(u) \beta(u) c\left[c^{-1} h \nu\right. & \left.=-\gamma\left(v_{\max }\right) m_{\mathrm{e}} v_{\max }+F \Delta t\right] \\
& + \\
\gamma(u)[h \nu & \left.=\left[\gamma\left(v_{\max }\right)-1\right] m_{\mathrm{e}} c^{2}+\Phi\right] .
\end{aligned}\right.
$$

Thus, the energy equation in the reference frame $\mathrm{S}^{\prime}$ can be expressed as a linear combination of the linear momentum equation and the energy equation in reference frame S.

A similar linear combination also apllies to the linear momentum, equation (37), which can be expressed as $\gamma(u) c \times$ Eq. (18) $+\gamma(u) \beta(u) \times$ Eq. (19), namely

$$
\left\{\begin{aligned}
\gamma(u) c\left[c^{-1} h \nu\right. & \left.=-\gamma\left(v_{\max }\right) m_{\mathrm{e}} v_{\max }+F \Delta t\right] \\
& + \\
\gamma(u) \beta(u)[h \nu & \left.=\left[\gamma\left(v_{\max }\right)-1\right] m_{\mathrm{e}} c^{2}+\Phi\right] .
\end{aligned}\right.
$$

Both the linear momentum and energy equations are needed to obtain a covariant description of the photoelectric effect.

For the electron braking process in $\mathrm{S}^{\prime}$, one has

$$
\mathrm{E}_{\mathrm{e}, \mathrm{f}}^{\prime}-\mathrm{E}_{\mathrm{e}, \mathrm{i}}^{\prime}=\mathrm{W}^{\prime}
$$

The energy equation for this process in $\mathrm{S}^{\prime}$ is

$$
\gamma(u) m_{\mathrm{e}} c^{2}-\gamma(u) \gamma\left(v_{\max }\right)\left[1-\beta(u) v_{\max } / c^{2}\right] m_{\mathrm{e}} c^{2}=-e \mathcal{E}_{\min } \gamma(u)\left[L-c \beta(u) t_{0}\right] \text {. }
$$

Equation (42) can be expressed as $-\gamma(u) \beta(u) c \times$ Eq. (23) $+\gamma(u) \times$ Eq. (24), namely

$$
\left\{\begin{aligned}
-\gamma(u) \beta(u) c\left[e \mathcal{E}_{\min } t_{0}\right. & \left.=\gamma\left(v_{\max }\right) m_{\mathrm{e}} v_{\max }\right] \\
& + \\
\gamma(u)\left[e \mathcal{E}_{\min } L\right. & \left.=\left[\gamma\left(v_{\max }\right)-1\right] m_{\mathrm{e}} c^{2}\right] .
\end{aligned}\right.
$$

Thus the energy equation in $\mathrm{S}^{\prime}$ can be expressed as a linear combination of the energy and momentum equations in $\mathrm{S}$. The linear momentum equation for this process in $\mathrm{S}^{\prime}$ is

$$
\gamma(u) \beta(u) m_{\mathrm{e}} c^{2}+\gamma(u) \gamma\left(v_{\max }\right)\left[v_{\max }-c \beta(u)\right] m_{\mathrm{e}} c=e \gamma(u) \mathcal{E}_{\min } \gamma(u)\left[c t_{0}-\beta(u) L\right] .
$$

Equation (44) can be expressed as $\gamma(u) c \times$ Eq. $(23)-\gamma(u) \beta(u) \times$ Eq. (24), namely

$$
\left\{\begin{aligned}
\gamma(u) c\left[e \mathcal{E}_{\min } t_{0}\right. & \left.=\gamma\left(v_{\max }\right) m_{\mathrm{e}} v_{\max }\right] \\
& + \\
-\gamma(u) \beta(u)\left[e \mathcal{E}_{\min } L\right. & \left.=\left[\gamma\left(v_{\max }\right)-1\right] m_{\mathrm{e}} c^{2}\right]
\end{aligned}\right.
$$


The linear momentum equation in $\mathrm{S}^{\prime}$ is a linear combination of linear momentum and energy equations in $\mathrm{S}$.

Of course, energy and linear momentum equations in the reference frame $\mathrm{S}$ can be expressed as the same linear combination, of the linear momentum and energy equations in reference frame $\mathrm{S}^{\prime}$ (except that $\vec{u}$ must be changed to $-\vec{u}$ ), according to the principle of relativity. 31

\section{CONCLUSIONS}

The analysis of the photoelectric effect is well-known. However, it is not generally noted that its explanation is a mixture of classical mechanics (kinetic energy of the electron), quantum physics (photon energy), and thermodynamics (work function as internal energy). The equations ordinarily used to describe the process are not covariant under Lorentz or Galilean transformations. In contrast, the relativistic description presented here is inherently covariant and leads one to consistently look at the process from a richer physical perspective.

The four-vector description of physical quantities, the equations between those fourvectors, and the transformation of the equations from one inertial reference frame to another, in a covariant description, results in this problem really being a little gem.

* guemezj@unican.es

$\dagger$ tmanuel@uc.pt

1 R. Wolfson, Essential University Physics, Vol. 2, 2nd Ed. (Addison-Wesley, San Francisco 2012), pp. 612-614.

2 R. A. Powell, "Photoelectric effect: Back to basics," Am. J. Phys. 46, 1046-1051 (1978).

3 H. D. Young, R. A. Freedman, and A. L. Ford, Sears and Zemansky's University Physics with Modern Physics, 13th ed. (Wiley, San Francisco, 2012), pp. 1261-1265.

4 R. N. Steinberg, G. E Oberem, and L. C. McDermott, "Development of a computer-based tutorial on the photoelectric effect," Am. J. Phys. 64, 1370-1379 (1996).

5 Mervi A. Asikainen, and Pekka E. Hirvonen, "A study of pre- and inservice teachers' understanding of photoelectric phenomenon as part of the development of a research-based quantum 
physics course," Am. J. Phys. 77, 658-666 (2009).

6 T. R. Carver, and J. O. Crawford, "Useful lecture demonstration of photoelectric wavelength theshold," Am. J. Phys. 43, 370-371 (1975).

7 A. Cortel, "Simple photoelectric effect," Phys. Teach. 44, 310 (2006).

8 R. J. Hanson, and B. E. Clotfelter, "Evaluation of commercial apparatus for measuring $h / e$," Am. J. Phys. 34, 75-78 (1966).

9 A. W. Knudsen, "A photoelectric determination of $h / e$ : A new approach to an old problem," Am. J. Phys. 51, 725-729 (1983).

10 A. Ahlgren, "APPARATUS FOR TEACHING PHYSICS: Inexpensive apparatus for studying the photoelectric effect and measuring Planck's constant," Phys. Teach. 1, 183-186 (1963).

11 J. Walker, Halladay and Resnick Principles of Physics. International Student Version, 9th ed. (Wiley, Hoboken, 2011), pp. 1059-1064.

12 H. H. Hall and R. P. Tuttle, "Photoelectric effect and Planck's constant in the introductory laboratory," Am. J. Phys. 39, 50-54 (1971).

13 R. A Serway, and J. W. Jewett, Jr., Principles of Physics. A calculus-Based Text, 3rd Ed. (Harcourt College Publisers, Orlando 2002), pp. 1049-1056.

14 M. Iona, "Stopping potential for photoelectrons," Am. J. Phys. 34, 707 (1966).

15 P. Gluck, and B. Saering, "No time lag in the photoelectric effect," Phys. Teach. 28, 285-286 (2010).

16 H. D. Young, R. A. Freedman, and A. L. Ford, Sears and Zemansky's University Physics with Modern Physics, 13th ed. (Wiley, San Francisco, 2012), p. 1263.

17 https://www.nobelprize.org/nobel_prizes/physics/laureates/

18 J Rudnick, and D. S. Tannhauser, "Concerning a widespread error in the description of the photoelectric effect," Am. J. Phys. 44, 796-798 (1976).

19 H. D. Young, R. A. Freedman, and A. L. Ford, Sears and Zemansky's University Physics with Modern Physics, 13th ed. (Wiley, San Francisco, 2012), p. 1264.

20 A. B. Arons and M. B. Peppard, "Einstein's proposal of the photon concept-a translation of the Annalen del Physics paper of 1905," Am. J. Phys. 33, 367-374 (1965).

21 R. Kidd, J. Ardini, and A. Anton, "Evolution of the modern photon," Am. J. Phys. 57, 27-35 (1989).

22 R. A. Millikan, "A direct photoelectric determination of Planck's ' $h$ '," Phys. Rev. 7, 355-388 
(1916).

23 D. R. Lloyd, "What was measured in Millikan's study of the photoelectric effect?," Am. J. Phys. 83, 765-772 (2015).

24 R. Baierlein, Newton to Einstein: The Trail of Light, (Cambridge University Press, New York 2002), pp. 151-154.

25 G. Margaritondo, "A historically correct didactic first step in the quantum world: stressing the interplay of relativity, thermodynamics and quantum physics," Eur. J. Phys. 24, 15-19 (2003).

26 R. Chabay and B. Sherwood, Matter and Interactions. I. Modern Mechanics, (Wiley, New York, 2002). pp. 1054-1057.

27 H. Hall and J. R. Oppenheimer, "Relativistic theory of the photoelectric effect," Phys. Rev. 38, 57-79 (1931)

28 H. Hall, "On the relativistic photoelectric effect," Phys. Rev. 45, 216-217 (1933)

29 J. Güémez, "An undergraduate exercise in the first law of relativistic thermodynamics," Eur. J. Phys. 31, 1209-1232 (2010).

30 J. Freund, Special Relativity for Beginners, (World Scientific, Singapore, 2008). Chap. 34.

31 J. Güémez, M. Fiolhais and L. A. Fernández, "Relativistic mechanical-thermodynamical formalism-description of inelastic collisions," Eur. J. Phys. 37, 015602 (21pp) (2015). 